\title{
Case Report: Diagnostic Challenges in the Detection of a Mixed Plasmodium vivax/ovale Infection in a Non-Endemic Setting
}

\author{
Ha Thu Trang Nguyen, ${ }^{1}$ Fabrizio Romano, ${ }^{2}$ Rahel Wampfler, ${ }^{3,4}$ Konrad Mühlethaler, ${ }^{1}$ Egbert Tannich, ${ }^{5}$ and Alexander Oberli ${ }^{1 *}$ \\ ${ }^{1}$ Institute for Infectious Diseases, University of Bern, Bern, Switzerland; ${ }^{2}$ Pediatric Emergency Department, Inselspital, Bern University Hospital, \\ University of Bern, Bern, Switzerland; ${ }^{3}$ Swiss Tropical and Public Health Institute, Basel, Switzerland; ${ }^{4}$ University of Basel, Basel, Switzerland; \\ ${ }^{5}$ Bernhard Nocht Institute for Tropical Medicine, Hamburg, Germany
}

\begin{abstract}
In clinical practice, mixed-species malaria infections are often not detected by light microscopy (LM) or rapid diagnostic test, as a low number of parasites of one species may occur. Here, we report the case of an 8-year-old girl migrating with her family from Afghanistan with a two-species mixed infection with Plasmodium vivax and Plasmodium ovale. This case demonstrates the significance of molecular testing in the detection of mixed-species malaria infections and highlights the importance of a detailed data analysis during the medical validation procedure to prevent underestimation of mixed-species infections. To our knowledge, this is the first case report of a two-species mixed infection comprising both $P$. vivax and $P$. ovale confirmed by LM and different real-time polymerase chain reaction (PCR) approaches.
\end{abstract}

\section{CASE}

Six months after immigrating to Switzerland, four members of a seven-member Afghan family (aged 2, 8, 10, and 15 years) were admitted to the pediatric emergency ward with a history of intermittent fever and malaise. Since their arrival in Switzerland, all four children have had repeated fever attacks, initially every 1-2 weeks. Further consultations followed because of increases in fever frequency, at the time of presentation every 2-3 days, accompanied by headache, nausea, and musculoskeletal and abdominal pain. In the past, all siblings were already treated for malaria in Dschalalabad, Afghanistan. Detailed information about diagnosis, prescribed antimalarial drugs, point of time, and duration of therapy could not be determined. On examination, all children presented in maintained general condition. No fever focus could be identified, and no organomegaly was palpable. The demographic and clinical laboratory data of each of the four children are summarized in Table 1. The reoccurring fever symptoms and the partially reduced thrombocyte counts were compatible with a relapsing or recrudescent form of malaria. Examination of peripheral blood smears by light microscopy (LM) according to standard protocols showed Plasmodium vivax-infected erythrocytes for all four siblings, with 1.05-3.25\% parasitemia, and rapid malaria antigen tests (BinaxNOW Malaria Test, Alere, Scarborough, ME) were all negative for Plasmodium falciparum and positive for pan-malaria antigen. For sibling 2, the LM revealed a $P$. vivax infection, but some schizonts showed features not typical for $P$. vivax. Very few schizonts comprised only eight merozoites, and some of the infected erythrocytes were oval, fimbriated, and slightly enlarged, thus resembling Plasmodium ovale schizonts (Figure $1 \mathrm{~A}$ and $\mathrm{B}$ ). However, the vast majority of schizonts were identified as $P$. vivax (Figure $1 \mathrm{C}$ and D). Retrospective analysis of ethylenediaminetetraacetic acid (EDTA) blood of sibling 2 using a Plasmodium speciesspecific multiplex real-time PCR (Fast Track Diagnostics (FTD) Malaria Differentiation, Fast Track Diagnostics, Sliema, Malta) revealed a $P$. vivax infection with a cycle threshold (Ct) value of 11.6. Subsequent in-detail analysis of the real-time PCR quantification data further showed an exponential/sigmoidal

*Address correspondence to Alexander Oberli, Parasitology Unit, Institute for Infectious Diseases, Friedbühlstrasse 51, Bern CH 3010 , Switzerland. E-mail: alexander.oberli@ifik.unibe.ch amplification curve for $P$. ovale, but the fluorescent signal was weak and below the minimum relative fluorescent units (RFU) peak height. To further investigate whether sibling 2 has $P$. vivax/P. ovale mixed infection or a single species infection, multiplex real-time PCR and monoplex real-time PCR with species-specific oligonucleotides were performed in the malaria reference laboratories of Switzerland (Swiss TPH) and Germany (Bernhard Nocht Institute for Tropical Medicine, Hamburg, Germany). Amplification of both $P$. vivax and $P$. ovale DNA by monoplex real-time PCR clearly indicated a mixed infection with $P$. vivax (Ct values 18.6 and 12.0) as the main species and $P$. ovale (Ct values 33.6 and 29.0) as the minor species. Subsequent characterization of the $P$. ovale subspecies by $P$. ovale-specific PCRs, based on a conserved region between $P$. ovale curtisi and $P$. ovale wallikeri, followed by sequencing of the amplified DNA, revealed sibling 2 being positive for $P$. ovale curtisi. ${ }^{1}$ All patients were initially treated with artemether/lumefantrine for 3 days (weight-based dose according to the international guidelines) followed by primaquine $0.5 \mathrm{mg} / \mathrm{kg} / \mathrm{dose}$ and chloroquin $2.5 \mathrm{mg} / \mathrm{kg} / \mathrm{dose}$ both once daily for 14 days to eradicate possible hypnozoites and to prevent relapses. There was a complete recovery from clinical symptoms for all four siblings and a normalization of blood thrombocyte counts.

\section{DISCUSSION}

Several species of the genus Plasmodium are known to cause malaria in humans, and clinical courses and severity of the disease differ according to the species. Therefore, an accurate and fast laboratory diagnosis is critical to administration, management, and treatment of malaria patients. Plasmodium vivax is the major cause of malaria morbidity in Afghanistan, and primaquine is in the treatment policy of Afghanistan although there is limited access to effective radical cure therapy and G6PD testing. ${ }^{2}$ As in Afghanistan $P$. vivax has a long latency period before relapse, the diagnosis of malaria should be considered in all febrile migrants from this region. ${ }^{2}$ In non-endemic regions, first-line malaria diagnosis commonly consists of a combination of LM (thick and thin blood smears) and rapid diagnostic test (RDT). ${ }^{3}$ Although LM of Giemsa-stained blood films is reasonable, fast, and inexpensive, differentiation of Plasmodium species and quantification of parasitemia require well-trained personnel. The 
TABLE 1

Patient demographic and clinical laboratory data

\begin{tabular}{|c|c|c|c|c|}
\hline & Sibling 1 & Sibling 2 & Sibling 3 & Sibling 4 \\
\hline Age (years) & 2 & 8 & 10 & 15 \\
\hline Time of diagnosis (since arrival) (months) & 6 & 9 & 7 & 6 \\
\hline Parasitemia (\%) & 3.25 & 1.5 & 1.4 & 1.05 \\
\hline BinaxNow rapid diagnostic test & non-P. $f$. & non-P. $f$. & non-P. $f$. & non- $P . f$ \\
\hline Parasite identification (microscopic) & P. vivax & P. vivax (Plasmodium ovale) & P. vivax & P. vivax \\
\hline $\begin{array}{l}\text { FTD real-time PCR cycle threshold-value } \\
\text { (P. vivax) }\end{array}$ & 19.0 & 11.6 & 16.4 & 15.4 \\
\hline Hemoglobin (10-16 g/dL) & 8 & 11.9 & 1.9 & 10 \\
\hline Thrombocytes $\left(150-450 \times 103 / \mathrm{mm}^{3}\right)$ & 121 & 107 & 131 & 136 \\
\hline Leukocytes $\left(3.0-12.5 \times 103 / \mathrm{mm}^{3}\right)$ & 9.7 & 6.1 & 6.36 & 4.72 \\
\hline C-reactive protein $(<3 \mathrm{mg} / \mathrm{L})$ & 46 & 32 & 7 & 50 \\
\hline Alanine transaminase $(\mathrm{U} / \mathrm{L})$ & 21 & 19 & 24 & 14 \\
\hline
\end{tabular}

P. $f .=$ Plasmodium falciparum; $P$. vivax $=$ Plasmodium vivax .

limit of parasite detection (LoD) of LM using thick blood films has been estimated to be about 50 parasites per microliter, whereas RDTs revealed good sensitivity of at least 200 parasites per microliter for the detection of $P$. falciparum, but the sensitivity for other Plasmodium species is limited. ${ }^{4}$ However, for the detection of mixed infections with two or more Plasmodium species, the combination of malaria microscopy and RDTs for the initial diagnosis has a relatively low sensitivity. ${ }^{5}$ Therefore, mixed infections have a high risk of not being detected. This has several reasons: 1) the morphological species differentiation of early-stage parasites (ring forms) can be misleading; 2) the parasitemia of the less abundant species may be below the LoD of LM; 3) after the initial diagnosis of the abundant species, the low abundant species might be overseen; and 4) one species is absent in the blood sample persisting as hypnozoites in the liver (in the case of $P$. vivax and $P$. ovale). Recent reports using molecular methods in parasite detection indicated reduction in costs and therapeutic
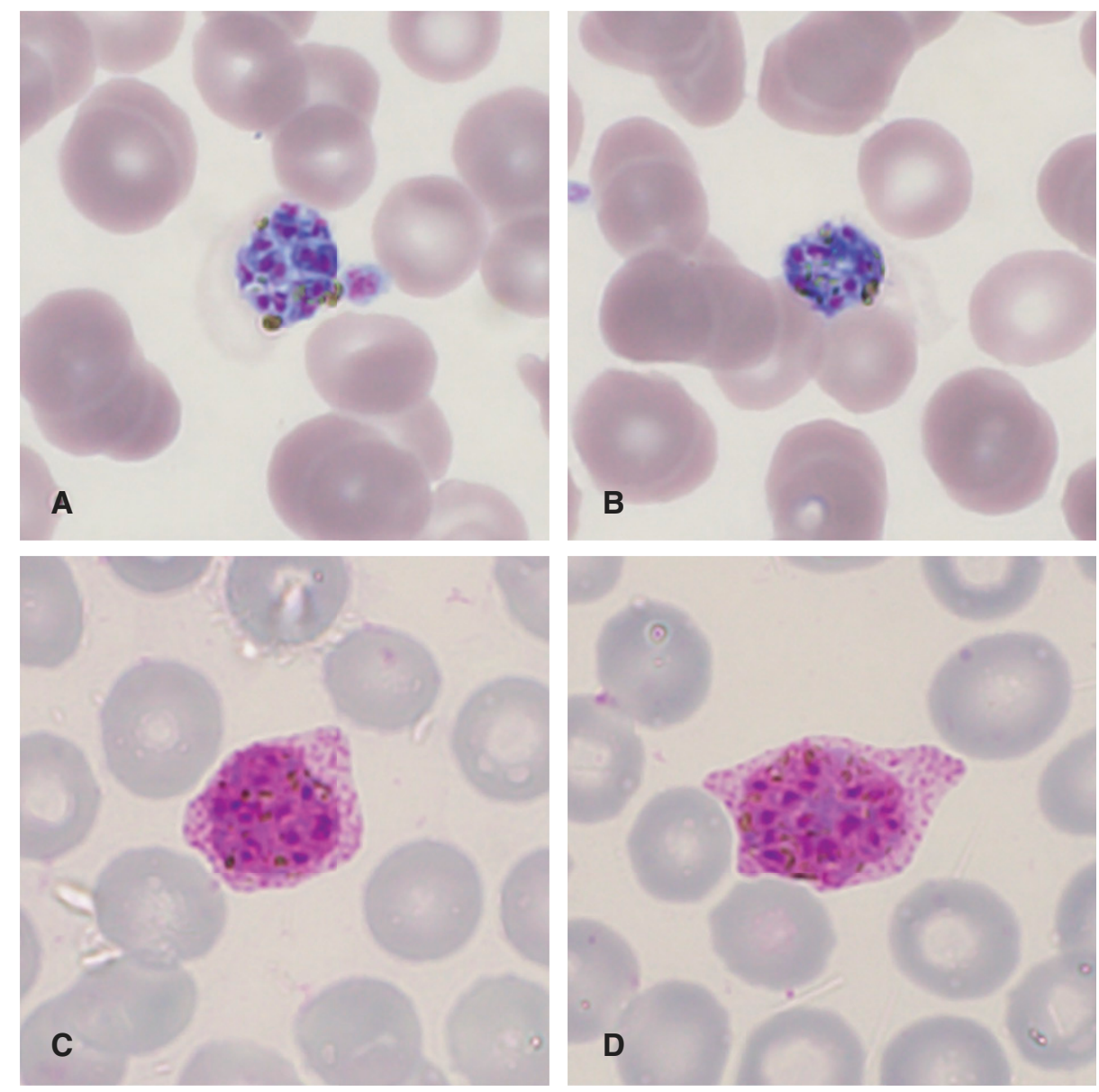

FIGURE 1. Light microscopy of a Giemsa-stained peripheral blood smear $(10 \times 100$ magnification $)$ of sibling 2. Besides characteristic Plasmodium vivax parasites $(\mathbf{C}+\mathbf{D})$, two mature schizonts with $13(\mathbf{A})$ and nine $(\mathbf{B})$ merozoites were observed. Note the fimbriated and oval-shaped infected erythrocytes with a cluster of dark brown pigment $(\mathbf{A}+\mathbf{B})$. This figure appears in color at www.ajtmh.org. 
turnaround time as well as higher prevalences of mixed-species infections compared with detection by LM. ${ }^{6,7}$ In the case of sibling 2, real-time PCR analysis confirmed a $P$. vivax infection with a high parasite load, as reflected by the relatively low Ct value. In-depth analysis of the melting curves revealed a fluorescent signal for $P$. ovale below the minimum RFU peak height. There is no standard rule for establishing minimum RFU thresholds for data interpretation, and this is one important aspect of the medical validation procedure of the data generated by the diagnostic laboratory. As there is no clear consensus on standardized PCR-based methods, diagnostic laboratories are strongly encouraged to follow the WHO external quality assurance scheme for malaria nucleic acid amplification testing. ${ }^{8}$ When the amount of DNA is very low, it is difficult to differentiate a true level RFU peak from signal noise or artifacts of any origin. Especially, in a multiplex real-time PCR setting with a mixed-species infection, RFU of the low abundant species might be suppressed. ${ }^{9}$ Subsequent monoplex real-time PCR with two different sets of $P$. ovale speciesspecific oligonucleotides confirmed a $P$. vivax/P. ovale mixed infection with a $P$. ovale curtisi ${ }^{1}$ subtype. To the best of our knowledge, this is the first documentation of a two-species mixed infection comprising both $P$. vivax and $P$. ovale detected by $L M$ and confirmed by real-time PCR. So far, mixed plasmodia infections comprising either $P$. vivax or $P$. ovale have only been described in combination with either $P$. falciparum and/or Plasmodium malariae as double- or triple-species infections. Moreover, the $P$. vivax/P. ovale species combination was only found in triple ( $P$. falciparum, $P$. vivax, and $P$. ovale) or quadruple infections ( $P$. falciparum, $P$. vivax, $P$. ovale, and $P$. malariae).$^{10,11}$ This might have different reasons. Plasmodium ovale may easily be confounded by other species, as usually parasitemia is lower than in $P$. falciparum or $P$. vivax infection. Moreover, species identification by LM requires experienced microscopists as the morphology of the two species resembles each other and, thus, may be difficult to differentiate in particular in cases of low parasitemia. Furthermore, it is discussed that simultaneous infection with different Plasmodium species can result in suppression of PCR for one of the species. ${ }^{9}$ Thus, it might be possible that $P$. ovale prevalence and parasitemia are consistently low for appropriate evaluation. Another explanation of the rarity of $P$. vivax/P. ovale mixed infection may be that these two species compete for invasion of host cells as both species prefer to invade reticulocytes. Because of the increased availability and increased use of species-specific malaria real-time PCR, it becomes evident that results obtained by LM are at least in part inaccurate, especially in cases of non-falciparum and mixed infections. A recent study combining $L M$ and real-time PCR revealed that molecular methods consistently detect higher prevalences for all malaria species infecting humans, with the highest increase in $P$. malariae and $P$. ovale. ${ }^{12}$ In all settings, in which molecular diagnostic tools such as real-time PCR are established and maintained, it is likely that real-time PCR will extend the diagnostic possibilities in case of incongruent LM and RDT not only to examine the presence of low-level parasitemia, but also to detect suspected mixedspecies infections, as it is a rapid, semiquantitative, sensitive, and specific diagnostic method to detect and differentiate malaria parasites. This is in accordance with a recent report, in which the good diagnostic performance of real-time PCR in non-endemic settings was confirmed and proposed to be included in the panel of diagnostic tools for imported malaria. ${ }^{13}$
This case highlights the importance of molecular testing and detailed data analysis in the medical validation process for the detection of a mixed-species malaria infection in a nonendemic region.

Received January 30, 2020. Accepted for publication March 10, 2020. Published online April 20, 2020.

Authors' addresses: Ha Thu Trang Nguyen, Konrad Mühlethaler, and Alexander Oberli, Institute for Infectious Diseases, University of Bern, Bern, Switzerland, E-mails: hathutrang.nguyen@ifik.unibe.ch, konrad.muehlethaler@ifik.unibe.ch, and alexander.oberli@ifik.unibe.ch. Fabrizio Romano, Pediatric Emergency Department, Inselspital, Bern University Hospital, University of Bern, Bern, Switzerland, E-mail: fabrizio.romano@insel.ch. Rahel Wampfler, Medical Parasitology and Infection Biology, Swiss Tropical and Public Health Institute, Basel, Switzerland, E-mail: rahel.wampfler@swisstph.ch. Egbert Tannich, Bernhard Nocht Institute for Tropical Medicine; Hamburg, Germany, E-mail: tannich@bnitm.de.

This is an open-access article distributed under the terms of the Creative Commons Attribution (CC-BY) License, which permits unrestricted use, distribution, and reproduction in any medium, provided the original author and source are credited.

\section{REFERENCES}

1. Fuehrer HP, Stadler MT, Buczolich K, Bloeschl I, Noedl H, 2012. Two techniques for simultaneous identification of Plasmodium ovale curtisi and Plasmodium ovale wallikeri by use of the smallsubunit rRNA gene. J Clin Microbiol 50: 4100-4102.

2. Leslie T, Hahzat S, Sediqi W, 2016. Epidemiology and control of Plasmodium vivax in Afghanistan. Am J Trop Med Hyg 95 (Suppl 6): 72-77.

3. Wongsrichanalai $\mathrm{CW}$, Barcus MJ, Muth S, Sutamihardja A, Wernsdorfer $\mathrm{WH}, 2007$. A review of malaria diagnostic tools: microscopic and rapid diagnostic test (RDT). Am J Trop Med Hyg 77: 119-127.

4. Chiodini PL, 2014. Malaria diagnostics: now and future. Parasitology 141: 1873-1879.

5. Mayxay M, Pukritrayakamee S, Chotivanich K, Imwong M, Looareesuwan S, White NJ, 2001. Identification of cryptic coinfection with Plasmodium falciparum in patients presenting with vivax malaria. Am J Trop Med Hyg 65: 588-592.

6. Hänscheid T, Grobusch MP, 2002. How useful is PCR in the diagnosis of malaria? Trends Parasitol 18: 395-398.

7. Ndao M, 2009. Diagnosis of parasitic diseases: old and new approaches. Interdiscip Perspect Infect Dis 2019: 278246.

8. World Health Organization, 2015. A WHO External Quality Assurance Scheme for Malaria Nucleic Acid Amplification Testing. Geneva, Switzerland: WHO.

9. Messerli C, Hofmann NE, Beck HP, Felger I, 2016. Critical evaluation of molecular monitoring in malaria drug efficacy trials and pitfalls of length-polymorphic markers. Antimicrob Agents Chemother 61: e01500-e01516.

10. Rubio JM, Benito J, Roche PJ, Berzosa ML, Garcia M, Mico M, Alvar J, 1999. Semi-nested, multiplex polymerase chain reaction for detection of human malaria parasites and evidence of Plasmodium vivax infection in Equatorial Guinea. Am J Trop Med Hyg 60: 183-187.

11. Oki M, Asai S, Saito-Nakano $\mathrm{Y}$, Nakayama T, Tanaka $\mathrm{Y}$, Tachibana H, Ohmae H, Nozaki T, Miyachi H, 2014. A case of quadruple malaria infection imported from Mozambique to Japan. Am J Trop Med Hyg 90: 1098-1101.

12. Tobian AA, Mehlotra RK, Malhotra I, Wamachi A, Mungai $P$, Koech D, Ouma J, Zimmerman P, King CL, 2000. Frequent umbilical cord-blood and maternal blood infections with Plasmodium falciparum, P. malariae, and $P$. ovale in Kenya. $J$ Infect Dis 182: 558-563.

13. Grossman T, Schwartz E, Vainer J, Agmon V, Glazer Y, Goldmann D, Marva E, 2017. Contribution of real-time PCR to Plasmodium species identification and to clinical decisions: a nationwide study in a non-endemic setting. Eur J Clin Microbiol Infect Dis 36: 671-675. 